03,11

\title{
Особенности распределения электронной плотности в теллуриде сурьмы $\mathrm{Sb}_{2} \mathrm{Te}_{3}$
}

\author{
() В.Г. Орлов ${ }^{1,2}$, Г.С. Сергеев ${ }^{1, \uparrow}$ \\ ${ }^{1}$ Национальный исследовательский центр „Курчатовский институт“, \\ Москва, Россия \\ ${ }^{2}$ Московский фризико-технический институт (Государственный университет), \\ Долгопрудный, Россия \\ I E-mail: Sergeev_GS@nrcki.ru
}

(Поступила в Редакцию 21 декабря 2016 г.)

На основе результатов расчетов электронной зонной структуры полупроводников $\mathrm{Sb}_{2} \mathrm{Te}_{3}, \mathrm{Ge}$, Tе и полуметалла $\mathrm{Sb}$, выполненных методом функционала электронной плотности, найдены параметры критических точек в распределении электронной плотности (максимумов, минимумов и седловых точек) в кристаллической решетке вышеуказанных веществ. Полученные данные использованы для анализа природы химической связи в $\mathrm{Sb}_{2} \mathrm{Te}_{3}$.

Работа выполнена при поддержке гранта РФФИ № 16-32-00107.

DOI: 10.21883/FTT.2017.07.44587.454

\section{1. Введение}

Теллурид сурьмы $\mathrm{Sb}_{2} \mathrm{Te}_{3}$ является узкозонным полупроводником, обладающим высокой термоэлектрической эффективностью [1]. В последние годы электронные свойства соединения $\mathrm{Sb}_{2} \mathrm{Te}_{3}$ привлекли особое внимание, поскольку первопринципные теоретические расчеты и результаты измерений методом фотоэлектронной спектроскопии с угловым разрешением (ARPES) показали, что $\mathrm{Sb}_{2} \mathrm{Te}_{3}$ обладает электронной структурой, в которой нижняя зона проводимости имеет одиночный дираковский конус. Это позволяет отнести $\mathrm{Sb}_{2} \mathrm{Te}_{3}$ к классу топологических изоляторов [2].

Кроме того, соединение $\mathrm{Sb}_{2} \mathrm{Te}_{3}$ принадлежит также к классу псевдобинарных сплавов $(\mathrm{GeTe})_{m}-\left(\mathrm{Sb}_{2} \mathrm{Te}_{3}\right)_{n}$, которые получили название материалов с изменяемым фазовым состоянием (phase change materials (PCM)) изза уникальной способности переходить из кристаллического состояния в аморфное и совершать обратный переход в кристаллическую фазу под действием лазерного излучения или импульса электрического тока за необычайно короткие времена порядка 1-100 ns. PCM в настоящее время используются для изготовления различных видов оптической перезаписываемой памяти компакт-дисков (CD), цифровых универсальных дисков (DVD), дисков на основе Blu-Ray технологии [3]. Высоко также оцениваются потенциальные возможности создания на основе РСМ энергонезависимой электронной памяти $[4,5]$.

Востребованность РСМ в практически важных приложениях для хранения данных обусловлена сочетанием их уникальных свойств: заметным различием оптических характеристик (коэффициентов отражения и пропускания лазерного света в широком спектральном диапазоне) в аморфном и кристаллическом состоянии [6], малыми временами и низким энергетическим порогом кристаллизации и аморфизации под действием лазерного излучения [7] или импульса электрического тока [8], резким увеличением электросопротивления при переходе из кристаллического в аморфное состояние $[8,9]$, стабильностью фазового состояния в области температур от комнатной до $150-200^{\circ} \mathrm{C}$ и способностью выдерживать большое число циклов перезаписи данных без заметного ухудшения свойств ячейки памяти [8].

Уникальность РСМ на основе $(\mathrm{GeTe})_{m}-\left(\mathrm{Sb}_{2} \mathrm{Te}_{3}\right)_{n}$ сплавов вызывает вопрос о связи рекордных параметров РСМ с особенностями их электронного строения. Признано, что выяснение природы химической связи в РСМ является ключевым моментом для понимания их свойств [5]. В ряде работ для описания кристаллических и аморфных полупроводников [10] и РСМ $[5,11]$ привлекалась идея о резонансной связи (resonance bonding), высказанная Полингом в его книге [12]. Основанная на квантовомеханических расчетах электронной структуры простейших двухатомных молекул, эта идея была, несомненно, полезна для качественного анализа свойств существенно более сложных многоатомных молекул. Но с появлением мощных ЭВМ и развитием численных методов расчетов электронной структуры молекул и конденсированных сред появилась возможность как качественного, так и количественного анализа влияния особенностей электронной структуры на свойства вещества.

В настоящей работе выполнены первопринципные расчеты электронной зонной структуры соединения $\mathrm{Sb}_{2} \mathrm{Te}_{3}$, а также веществ $\mathrm{Ge}$, Tе и $\mathrm{Sb}$, входящих в состав РСМ. На основе полученных результатов проведен анализ распределения электронной плотности в исследованных соединениях, позволяющий выявить наличие особенностей в электронной структуре, которые характерны для РСМ. 


\section{2. Метод расчета электронной зонной структуры и анализа распределения электронной плотности}

Наибольшую популярность в области численного моделирования электронных свойств молекул и конденсированных сред приобрел метод функционала электронной плотности (Density Functional Theory (DFT)), основанный на теоремах Хоэнберга-Кона [13] и формализме Кона-Шэма [14]. Метод DFT позволил свести сложную задачу о поведении большого числа взаимодействующих электронов, помещенных в кулоновский потенциал атомных ядер, к задаче о невзаимодействующем электронном газе, находящемся в эффективном потенциале, который учитывает как электростатический потенциал ядер, так и обменно-корреляционные эффекты в электронной подсистеме. При этом энергия электронной подсистемы является функционалом электронной плотности $\rho(\mathbf{r})$, зависящей только от радиус-вектора $\mathbf{r}$.

За время, прошедшее с момента публикации работ $[13,14]$, было создано несколько десятков программных пакетов для расчетов электронной структуры конденсированных сред, некоторые из которых (ABINIT, LMTO, CASTEP, VASP) были использованы для исследования механизма химической связи в РСМ, а также для установления корреляции между наличием вакансий и локальных искажений структуры в РСМ и процессами их кристаллизации и аморфизации [15-19]. Однако результаты расчетов электронной зонной структуры, дающие чрезвычайно полезные сведения, например о полной электронной энергии основного состояния вещества, и позволяющие сделать вывод о предпочтительности той или иной кристаллической структуры, сами по себе не предоставляют информацию о природе механизма химической связи в веществе на атомном уровне. Для наглядной визуализации роли электронной плотности в химической связи в РСМ авторы цитированных выше работ использовали такие методы как CDD (charge density difference - разность зарядовой плотности в веществе и в атомах, входящих в состав вещества) [15,17-19] и COHP (crystal orbital Hamilton population - представление энергии зонной структуры в виде вкладов от различных межатомных взаимодействий) [16].

Между тем в квантовой химии разработан и широко используется метод нахождения особенностей в распределении электронной плотности $\rho(\mathbf{r})$ молекул [20], основанный на анализе матрицы вторых производных электронной плотности по координатам, называемой матрицей Гессе, или гессианом

$$
\left(\partial^{2} \rho / \partial x_{i} \partial x_{j}\right)
$$

где $i, j=1,2,3, x_{1}=x, x_{2}=y, x_{3}=z$. При этом электронная плотность $\rho(\mathbf{r})$, которая находится либо из экспериментов, либо расчетным путем, рассматривается как скалярное поле. Точки $\mathbf{r}_{c}$, в которых градиент электронной плотности обращается в нуль $\nabla \rho\left(\mathbf{r}_{c}\right)=0$, называются критическими. В этих точках электронная плотность обладает экстремальными свойствами (имеет минимум или максимум, а также может быть седловой точкой). Диагонализуя симметричную матрицу Гессе $\rho$, можно найти ее собственные значения $\lambda_{i}(i=1,2,3)$ и координатные оси - главные оси кривизны, для которых недиагональные элементы матрицы равны нулю. Сумма диагональных матричных элементов - лапласиан $\nabla^{2} \rho=\partial^{2} \rho / \partial x^{2}+\partial^{2} \rho / \partial y^{2}+\partial^{2} \rho / \partial z^{2}$ выполняет важную роль в анализе особенностей в распределении электронной плотности. Знак лапласиана и его величина в критической точке служат одним из признаков типа химической связи [20]. Отрицательный знак лапласиана в критической точке $\nabla^{2} \rho_{b}<0$ свидетельствует о натекании заряда в критическую точку от атомов, между которыми данная критическая точка расположена. Напротив, положительный знак лапласиана $\nabla^{2} \rho_{b}>0$ говорит о выталкивании заряда из области вблизи критической точки. Собственные значения $\lambda_{i}$ симметричной матрицы Гессе $\rho$ (главные значения кривизны) действительны, могут иметь любой знак, а также равняться нулю. Классификация особых точек $\rho$ осуществляется по рангу $\omega$ - числу ненулевых главных значений кривизны и сигнатуре $\sigma-$ алгебраической сумме их знаков: $(\omega, \sigma)$. Критическая точка $(3,-3)$ отвечает локальному максимуму $\rho$. Несмотря на то что в местах расположения ядер атомов, входящих в молекулу, $\nabla \rho$ терпит разрыв, в равновесном состоянии молекул локальные максимумы электронной плотности $\rho$ на ядрах, называемые nucleus, рассматриваются как критические точки с параметрами $(3,-3)[20]$. Критическая точка $(3,+3)$ представляет собой локальный минимум в распределении электронной плотности и носит название клетки (cage). Из двух седловых критических точек $(3,+1)$-кольцо (ring) и $(3,-1)$-связь (bond); последняя, как следует из названия, играет важную роль в классификации типа химической связи.

Использование метода критических точек в распределении электронной плотности для анализа природы химической связи в кристаллах $[21,22]$ оказалось столь же востребованным, как и в химии молекул. В простейшем варианте классификации типов химической связи в кристаллах в качестве критериев рассматриваются знак лапласиана электронной плотности $\nabla^{2} \rho_{b}$ в седловой критической точке типа bond $(3,-1)$, знаки и соотношения абсолютных величин главных значений кривизны $\lambda_{i}(i=1,2,3)$ в данной критической точке, величина заряда $\rho_{b}$, а также характер распределения электронной плотности в межатомной области кристалла. В частности, ковалентная связь характеризуется отрицательным знаком лапласиана $\nabla^{2} \rho_{b}<0$, отрицательными значениями $\lambda_{1,2}<0$, большими по абсолютной величине $\left|\lambda_{1,2}\right|>\lambda_{3}$, большим значением электронной плотности в критической точке $\rho_{b}$. В то время как для ионной связи $\nabla^{2} \rho_{b}>0,\left|\lambda_{1,2}\right| \ll \lambda_{3}, \rho_{b}$ мало, зарядовая плотность концентрируется в основном в областях расположения атомов $[21,22]$. 
Таблица 1. Экспериментальные значения параметров решеток кристаллов, использованные в расчетах, теоретические и экспериментальные значения полупроводниковой щели

\begin{tabular}{|c|c|c|c|c|}
\hline & $\mathrm{Ge}$ & $\mathrm{Sb}$ & $\mathrm{Te}$ & $\mathrm{Sb}_{2} \mathrm{Te}_{3}$ \\
\hline $\begin{array}{c}\text { Параметры } \\
\text { решетки, } \\
\text { ссылки }\end{array}$ & $\begin{array}{c}a=5.658 \\
{[28]}\end{array}$ & $\begin{array}{l}\mathrm{a}_{\mathrm{h}}=4.308 \\
\mathrm{c}_{\mathrm{h}}=11.274 \\
\mathrm{a}_{\mathrm{rh}}=4.507 \\
\alpha=57.11^{\circ} \\
\quad[29]\end{array}$ & $\begin{array}{c}\mathrm{a}=4.456 \\
\mathrm{c}=5.923 \\
\\
\\
\quad[30]\end{array}$ & $\begin{array}{c}\mathrm{a}_{\mathrm{h}}=4.264 \\
\mathrm{c}_{\mathrm{h}}=30.458 \\
\mathrm{a}_{\mathrm{rh}}=10.447 \\
\alpha=23.55^{\circ} \\
{[31]}\end{array}$ \\
\hline $\begin{array}{c}\text { Пространственная } \\
\text { группа }\end{array}$ & $\begin{array}{c}227 \\
F d \overline{3} m\end{array}$ & $\begin{array}{c}166 \\
R \overline{3} m\end{array}$ & $\begin{array}{c}152 \mathrm{P} 3_{1} 21 \text { или } \\
153 \mathrm{P} 3_{2} 21 \\
\end{array}$ & $\begin{array}{r}166 \\
R \overline{3} m \\
\end{array}$ \\
\hline Щель, eV, расчет & 0.749 & Полуметалл & 0.478 & 0.148 \\
\hline $\begin{array}{c}\text { Щель, eV, } \\
\text { эксперимент } \\
\text { ссылки }\end{array}$ & $\begin{array}{l}0.74 \\
{[32]}\end{array}$ & - & $\begin{array}{c}0.335 \\
{[33]}\end{array}$ & $\begin{array}{l}0.21 \\
{[34]}\end{array}$ \\
\hline
\end{tabular}

В настоящей работе с помощью пакета программ WIEN2k (версия 14.2) [23] выполнены расчеты электронной зонной структуры соединения $\mathrm{Sb}_{2} \mathrm{Te}_{3}$, принадлежащего классу псевдобинарных сплавов $(\mathrm{GeTe})_{m}-\left(\mathrm{Sb}_{2} \mathrm{Te}_{3}\right)_{n}$, обладающих свойствами РСМ, a также кристаллов полупроводников $\mathrm{Ge}$, Те и полуметалла Sb. Вычисления проводились методом FPLAPW (Full Potential Linearized Augmented Plane Wave) c использованием модифицированного Траном и Блахой обменно-корреляционного потенциала Беке и Джонсона [24] (mBJ - modified Becke-Johnson exchange potential + LDA-correlation), который позволяет получать корректные значения диэлектрической щели в полупроводниках и оксидах [25]. Также учитывалось спинорбитальное взаимодействие и релятивистские поправки. Для обеспечения необходимой точности при расчетах использовались следующие параметры: разложение по $l$-орбитальному моменту проводилось внутри атомных сфер до $l_{\max }=10$, радиусы атомных сфер $R_{\min }$ брались равными 2.3,2.1 и 2.3 at.units для $\mathrm{Ge}, \mathrm{Te}$ и $\mathrm{Sb}$ соответственно. Максимальное значение волнового вектора $k_{\max }$, использующееся при разложении по плоским волнам, определялось условием $R_{\min } \cdot k_{\max }=8.0$, общее число точек в зоне Бриллюэна выбиралось равным 2000 для всех исследуемых веществ.

Вычисления проводились на многоцелевом вычислительном комплексе НИЦ „Курчатовский институт“ $[26]$.

Поиск критических точек в распределении электронной плотности, полученной с помощью программного комплекса WIEN2k, проводился на основе программы Critic 2 [27], предназначенной для топологического анализа скалярных полей в периодических структурах.

В расчетах параметры кристаллических решеток веществ брались из экспериментальных данных, приведенных в табл. 1. Указанные в табл. 1 параметры решетки были измерены при комнатной температуре. Для кристаллов $\mathrm{Sb}$ и $\mathrm{Sb}_{2} \mathrm{Te}_{3}$, имеющих ромбоэдрическую симметрию, параметры решеток даны в двух вариан- тах осей - гексагональных $\left(\mathrm{a}_{\mathrm{h}}, \mathrm{c}_{\mathrm{h}}\right)$ и ромбоэдрических $\left(\mathrm{a}_{\mathrm{rh}}, \alpha\right)$. В табл. 1 также приведены экспериментальные и рассчитанные в настоящей работе величины полупроводниковых щелей.

\section{3. Результаты и обсуждение}

Результаты поиска критических точек в распределении электронной плотности кристаллов $\mathrm{Ge}, \mathrm{Sb}, \mathrm{Te}$, а также соединения $\mathrm{Sb}_{2} \mathrm{Te}_{3}$ приведены в табл. 2. В частности, в табл. 2 дана информация о типах критических точек, их количестве $N_{c}$ и типе позиций высокой симметрии, которые занимают критические точки в решетке кристалла. Также в табл. 2 указаны координаты $(x, y, z$ в долях от параметров решетки) одной из критических точек каждого типа, величины главных значений кривизны $\lambda_{i}(i=1,2,3)$ распределения электронной плотности в данной критической точке, значения лапласиана $\nabla^{2} \rho_{c}$ и значение $\rho_{c}$ в критической точке. У веществ со структурами, описываемыми пространственной группой № 166 $R \overline{3} m$, координаты критических точек приведены в долях от параметра решетки в ромбоэдрических осях $a_{r h}$. Для критических точек типа nucleus параметры не приведены в силу их нефизичности.

Пространственно-групповая симметрия накладывает ограничения на типы и позиции критических точек. У всех исследованных нами веществ критические точки располагаются в позициях высокой симметрии пространственных групп кристаллов [35], указанных в табл. 1. При этом количество критических точек в элементарной ячейке кристаллов удовлетворяет соотношению Морca $[21,22,36]$

$$
n-b+r-c=0 ; \quad c \geq 1, \quad r \geq 3, \quad b \geq 3, \quad n \geq 1,
$$

где $n, b, r$ и $c$ - количество критических точек типа nucleus, bond, ring и cage соответственно. 
Таблица 2. Параметры критических точек распределения электронной плотности в кристаллах $\mathrm{Ge}, \mathrm{Sb}, \mathrm{Te}_{\text {и }} \mathrm{Sb}_{2} \mathrm{Te}_{3}$

\begin{tabular}{|c|c|c|c|c|c|c|c|c|c|c|}
\hline & $\begin{array}{c}\text { Тип критической } \\
\text { точки }\end{array}$ & $\begin{array}{l}N_{c}, \text { тип } \\
\text { позиции }\end{array}$ & $\mathrm{x}$ & $\mathrm{y}$ & z & $\lambda_{1}, \mathrm{e} / \AA^{5}$ & $\lambda_{2}, \mathrm{e} / \AA^{5}$ & $\lambda_{3}, \mathrm{e} / \AA^{5}$ & $\nabla^{2} \rho_{c}, \mathrm{e} / \AA^{5}$ & $\rho_{c}, \mathrm{e} / \AA^{3}$ \\
\hline $\mathrm{Ge}$ & $\begin{array}{l}\text { nucleus } \\
\text { bond } \\
\text { ring } \\
\text { cage }\end{array}$ & $\begin{array}{c}8 \mathrm{a} \\
16 \mathrm{c} \\
16 \mathrm{~d} \\
8 \mathrm{~b}\end{array}$ & $\begin{array}{c}0.0 \\
0.875 \\
0.625 \\
0.500\end{array}$ & $\begin{array}{c}0.0 \\
0.875 \\
0.875 \\
0.000\end{array}$ & $\begin{array}{c}0.0 \\
0.125 \\
0.875 \\
0.000\end{array}$ & $\begin{array}{c}- \\
-1.581 \\
-0.039 \\
0.054\end{array}$ & $\begin{array}{r}- \\
-1.581 \\
0.153 \\
0.054\end{array}$ & $\begin{array}{c}- \\
0.914 \\
0.153 \\
0.054\end{array}$ & $\begin{array}{c}- \\
-2.248 \\
0.267 \\
0.162\end{array}$ & $\begin{array}{c}- \\
0.549 \\
0.026 \\
0.014\end{array}$ \\
\hline $\mathrm{Sb}$ & $\begin{array}{c}\text { nucleus } \\
\text { bond } \\
\gg \\
\text { ring } \\
\text { cage } \\
\gg\end{array}$ & $\begin{array}{l}2 \mathrm{c} \\
3 \mathrm{~d} \\
3 \mathrm{e} \\
6 \mathrm{~h} \\
1 \mathrm{~b} \\
1 \mathrm{a}\end{array}$ & $\begin{array}{l}0.234 \\
0.000 \\
0.500 \\
0.748 \\
0.500 \\
0.000\end{array}$ & $\begin{array}{l}0.234 \\
0.000 \\
0.500 \\
0.300 \\
0.500 \\
0.000\end{array}$ & $\begin{array}{l}0.234 \\
0.500 \\
0.000 \\
0.748 \\
0.500 \\
0.000\end{array}$ & $\begin{array}{r}- \\
-0.907 \\
-0.359 \\
-0.090 \\
0.015 \\
0.045\end{array}$ & $\begin{array}{c}- \\
-0.890 \\
-0.349 \\
0.235 \\
0.090 \\
0.045\end{array}$ & $\begin{array}{c}- \\
1.327 \\
1.174 \\
0.248 \\
0.090 \\
0.086\end{array}$ & $\begin{array}{c}- \\
-0.469 \\
0.466 \\
0.394 \\
0.196 \\
0.176\end{array}$ & $\begin{array}{c}- \\
0.372 \\
0.167 \\
0.053 \\
0.021 \\
0.018\end{array}$ \\
\hline $\mathrm{Te}$ & $\begin{array}{c}\text { nucleus } \\
\text { bond } \\
\gg \\
\text { ring } \\
\gg \\
\text { cage }\end{array}$ & $\begin{array}{l}3 \mathrm{a} \\
3 \mathrm{~b} \\
6 \mathrm{c} \\
3 \mathrm{a} \\
6 \mathrm{c} \\
3 \mathrm{~b}\end{array}$ & $\begin{array}{l}0.263 \\
0.128 \\
0.124 \\
0.254 \\
0.464 \\
0.627\end{array}$ & $\begin{array}{l}0.000 \\
0.128 \\
0.619 \\
0.254 \\
0.789 \\
0.627\end{array}$ & $\begin{array}{l}0.333 \\
0.500 \\
0.506 \\
1.000 \\
0.636 \\
0.500\end{array}$ & $\begin{array}{r}- \\
-1.140 \\
-0.317 \\
-0.071 \\
-0.059 \\
0.006\end{array}$ & $\begin{array}{c}- \\
-1.081 \\
-0.291 \\
0.218 \\
0.089 \\
0.041\end{array}$ & $\begin{array}{c}- \\
1.900 \\
1.240 \\
0.251 \\
0.263 \\
0.090\end{array}$ & $\begin{array}{c}- \\
-0.320 \\
0.632 \\
0.398 \\
0.294 \\
0.137\end{array}$ & $\begin{array}{c}- \\
0.435 \\
0.140 \\
0.042 \\
0.035 \\
0.013\end{array}$ \\
\hline $\mathrm{Sb}_{2} \mathrm{Te}_{3}$ & $\begin{array}{c}\text { nucleus } \\
\gg \\
\gg \\
\text { bond } \\
\gg \\
\gg \\
\text { ring } \\
\gg \\
\gg \\
\text { cage } \\
\gg \\
\gg\end{array}$ & $\begin{array}{l}1 \mathrm{a} \\
2 \mathrm{c} \\
2 \mathrm{c} \\
6 \mathrm{~h} \\
6 \mathrm{~h} \\
3 \mathrm{~d} \\
6 \mathrm{~h} \\
3 \mathrm{e} \\
6 \mathrm{~h} \\
2 \mathrm{c} \\
2 \mathrm{c} \\
1 \mathrm{~b}\end{array}$ & $\begin{array}{l}0.00 \\
0.211 \\
0.400 \\
0.416 \\
0.208 \\
0.500 \\
0.618 \\
0.500 \\
0.276 \\
0.096 \\
0.302 \\
0.500\end{array}$ & $\begin{array}{l}0.000 \\
0.211 \\
0.400 \\
0.890 \\
0.208 \\
0.000 \\
0.099 \\
0.500 \\
0.875 \\
0.096 \\
0.302 \\
0.500\end{array}$ & $\begin{array}{c}0.00 \\
0.211 \\
0.400 \\
0.416 \\
0.687 \\
0.000 \\
0.099 \\
1.000 \\
0.276 \\
0.096 \\
0.302 \\
0.500\end{array}$ & $\begin{array}{c}- \\
- \\
- \\
-0.882 \\
-0.564 \\
-0.206 \\
-0.106 \\
-0.092 \\
-0.056 \\
0.012 \\
0.042 \\
0.032\end{array}$ & $\begin{array}{c}- \\
- \\
- \\
-0.878 \\
-0.553 \\
-0.202 \\
0.248 \\
0.094 \\
0.137 \\
0.090 \\
0.069 \\
0.035\end{array}$ & $\begin{array}{c}- \\
- \\
- \\
1.788 \\
1.580 \\
0.990 \\
0.318 \\
0.411 \\
0.230 \\
0.090 \\
0.069 \\
0.035\end{array}$ & $\begin{array}{c}- \\
- \\
- \\
0.029 \\
0.463 \\
0.582 \\
0.460 \\
0.413 \\
0.311 \\
0.193 \\
0.179 \\
0.102\end{array}$ & $\begin{array}{c}- \\
- \\
- \\
0.342 \\
0.234 \\
0.103 \\
0.058 \\
0.053 \\
0.035 \\
0.020 \\
0.018 \\
0.010\end{array}$ \\
\hline
\end{tabular}

Из табл. 2 следует, что знаки и величины главных значений кривизны $\lambda_{1}, \lambda_{2}, \lambda_{3}$, лапласиана $\nabla^{2} \rho_{b}$ и электронной плотности $\rho_{b}$ в критических точках типа bond в $\mathrm{Ge}$, в соответствии с простейшей классификацией типов химической связи [21,22], подтверждают общепринятое представление о $\mathrm{Ge}$ как о соединении с высокой степенью ковалентности.

Несмотря на то что лапласиан электронной плотности отрицателен $\nabla^{2} \rho_{b}<0$ в критических точках bond первого типа, занимающих $d$-позиции в кристаллической структуре $\mathrm{Sb}$, соотношения между абсолютными величинами главных значений кривизны $\left|\lambda_{1,2}\right|<\lambda_{3}$ для данных критических точек не удовлетворяют условиям простейшей классификации, как для ковалентного, так и для ионного типов связи в кристалле [21,22]. У критических точек bond второго типа, находящихся в $e$-позициях кристаллической структуры $\mathrm{Sb}, \nabla^{2} \rho_{b}>0$, а соотношения между абсолютными величинами главных значений кривизны такие же, как у критических точек bond первого типа. Данные параметры главных значений кривизны распределения электронной плотности в критических точках типа bond в кристалле $\mathrm{Sb}$, а также знаки и значения лапласиана $\nabla^{2} \rho_{b}>0$ могут рассматриваться как характерные для полуметалла.

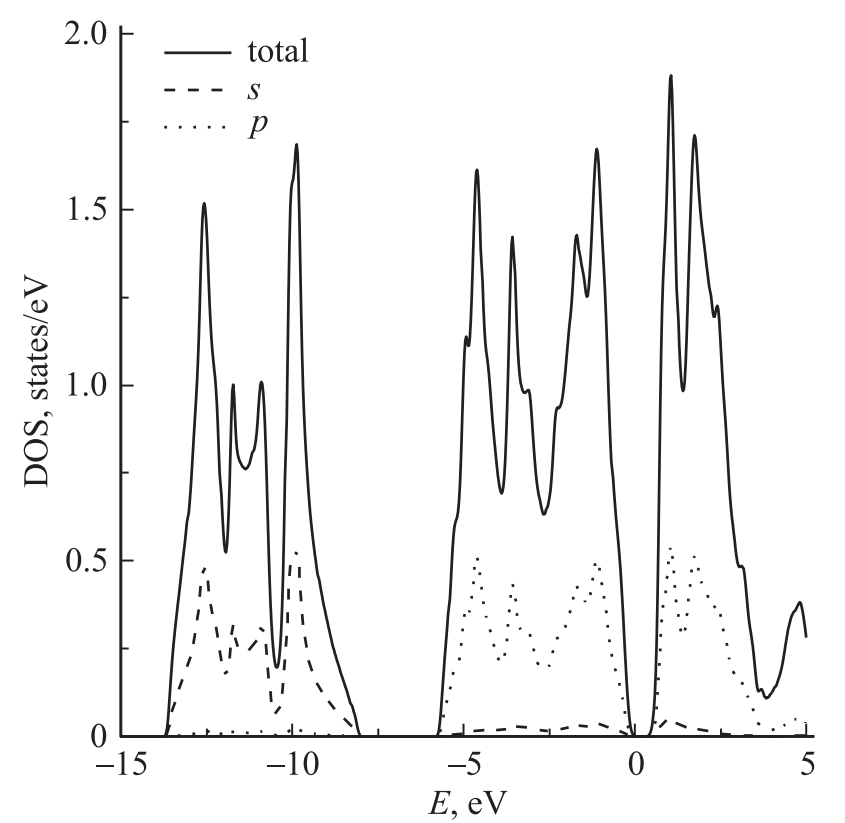

Рис. 1. Парциальные плотности состояний $s$ - и $p$-электронов на один атом, а также полная плотность электронных состояний в ячейке кристалла Те. 

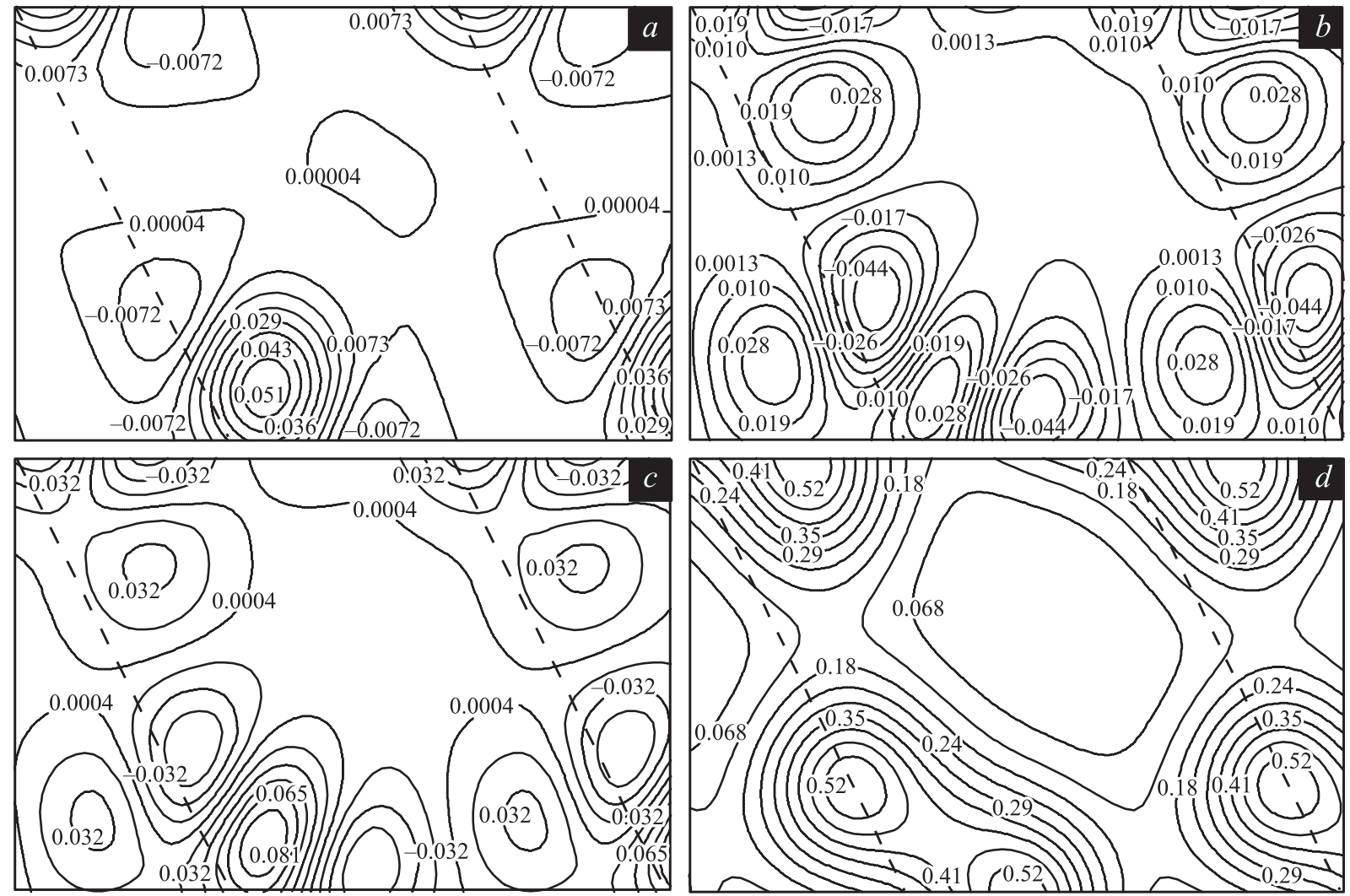

Pис. 2. Линии уровня распределения электронной плотности в плоскости кристалла Те, содержащей критические точки типа bond и cage. $a$ - вклад $s$-электронов, $b-$ вклад $p$-электронов, $c-$ вклад $s$ - и $p$-электронов, $d-$ полная электронная плотность. Цифры около линий показывают значение электронной плотности на данной линии уровня в единицах е/ $\AA^{3}$.

На рис. 1 показаны парциальные и суммарная плотности электронных состояний $s$ - и $p$-электронов валентной полосы Те. Из данного рисунка следует, что с точностью до нескольких процентов вклады от $s$ - и $p$-электронов в электронную плотность Те можно разделить. В области энергий от 0 до $-6 \mathrm{eV}$, основной вклад в которую дают p-электроны Те, вследствие дисперсии зон, разделение их на две области - зоны связывающих $p$-электронов с энергией от -6 до $-3 \mathrm{eV}$ и область неподеленной пары (от -3 до $0 \mathrm{eV}$ ), которое предлагалось в ранних работах по расчетам электронной структуры Те (см., например, [37]), может быть выполнено лишь с существенной ошибкой. Вследствие этого, вклад валентных р-электронов в распределение электронной плотности вблизи критических точек типа bond в кристалле Те оценивался нами без разделения на подобласти.

На рис. 2 представлено распределение электронной плотности от $s$ - и $p$-электронов Те в кристаллической плоскости, которая параллельна плоскости $(a, b)$ системы координат и пересекает ось $c$ на половине высоты. В этой плоскости в пределах границы элементарной ячейки, обозначенной двумя наклонными пунктирными линиями, лежит одна из трех седловых критических точек bond первого типа с координатами $(0.128,0.128,0.5)$, которая находится на середине расстояния между двумя ближайшими атомами Те, принадлежащими одному витку спиральной структуры кристалла Те [30]. Вблизи данной плоскости находятся две из шести седловых критических точек bond второго типа с координатами $(0.124,0.619,0.506)$ и $(0.619,0.124,0.494)$, которые обеспечивают связь между спиралями из атомов Те. В данной плоскости также располагается одна из трех критических точек типа cage с координатами $(0.627,0.627,0.500)$. На рис. $2, d$ дано распределение полной электронной плотности от $s$ - и $p$-электронов Те в указанной плоскости, показанное с помощью линий уровня. Максимумы электронной плотности, между которыми находятся седловые критические точки bond первого и второго типа, происходят от атомов Те с координатами $(0.263,0.0,0.333)$ и $(0.0,0.263,0.667)$, которые располагаются ниже и выше плоскости рисунка. На рис. 2, $a-c$ изображены соответственно парциальные вклады от $s, p$ и суммарный вклад от $s$ - и $p$-электронов в электронную плотность седловых критических точек bond первого и второго типа, полученные методом CDD. Из рис. $2, a$ и $b$ следует, что в электронную плотность критической точки bond первого типа $s$-электроны Те дают больший вклад, чем $p$-электроны, в то время как электронная плотность в критических точках bond второго типа обусловлена, главным образом, вкладом р-электронов. В ранних работах по расчету электронной зонной структуры Те 


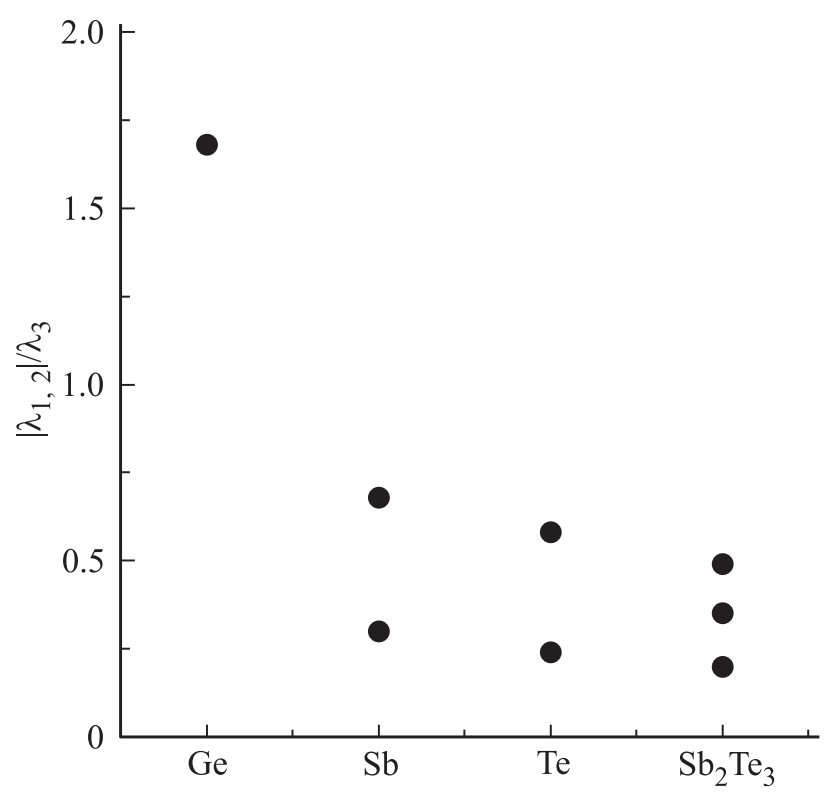

Рис. 3. Отношения главных значений кривизны $\left|\lambda_{1,2}\right|<\lambda_{3}$ в критических точках распределения электронной плотности типа bond для $\mathrm{Ge}, \mathrm{Sb}, \mathrm{Te}$ и $\mathrm{Sb}_{2} \mathrm{Te}_{3}$.

вкладом $s$-электронов в образование связи между атомами Те, находящимися внутри одной спирали, обычно пренебрегали [37].

Несмотря на то что Те является полупроводником, параметры лапласиана и значения электронной плотности в критических точках типа bond для Те близки к значениям соответствующих параметров у полуметалла $\mathrm{Sb}$.

Как следует из табл. 2, у всех трех критических точек типа bond в распределении электронной плотности в кристалле $\mathrm{Sb}_{2} \mathrm{Te}_{3}$ лапласиан положителен $\nabla^{2} \rho_{b}>0$ и соотношения между главными значениями кривизны $\left|\lambda_{1,2}\right|<\lambda_{3}$ аналогичны тем, которые были найдены для $\mathrm{Sb}$ и $\mathrm{Te}$.

Если пренебречь небольшой эллиптичностью в распределении электронной плотности вблизи критических точек типа bond в $\mathrm{Sb}, \mathrm{Te}$ и $\mathrm{Sb}_{2} \mathrm{Te}_{3}$ и приближенно считать, что $\left|\lambda_{1}\right| \approx\left|\lambda_{2}\right|$ для данных веществ, то можно использовать отношение $\left|\lambda_{1,2}\right| / \lambda_{3}$ для критических точек типа bond как некоторую характеристику степени ковалентности химической связи в веществе [20-22]. Из рис. 3, на котором представлено данное отношение для всех критических точек типа bond в исследованных нами веществах, следует, что химическая связь в соединении $\mathrm{Sb}_{2} \mathrm{Te}_{3}$ обладает наименьшей степенью ковалентности.

Кристаллическая структура $\mathrm{Sb}_{2} \mathrm{Te}_{3}$ имеет ромбоэдрическую симметрию с пятью атомами в элементарной ячейке [31]: атомы $\mathrm{Te}_{1}$ занимают позиции типа 1a, атомы $\mathrm{Te}_{2}$ и $\mathrm{Sb}$ - позиции типа 2c. Но структуру $\mathrm{Sb}_{2} \mathrm{Te}_{3}$ также часто (см., например, [38]) представляют в гексагональных осях в виде 15-слойной структуры, изображенной на рис. 4 и состоящей из трех наборов по пять слоев атомов в каждом (так называемые „quintuple layers of atoms“). Квинтет образуют слои с четвертого по восьмой и с девятого по тринадцатый. На рис. 4 показаны 3 набора ближайших расстояний между атомами в структуре $\mathrm{Sb}_{2} \mathrm{Te}_{3}$, представленные в виде линий, соединяющих атомы. Критические точки типа bond в распределении электронной плотности находятся между атомами: $\mathrm{Sb}-\mathrm{Te}_{2}$ (первый набор критических точек типа bond), $\mathrm{Sb}-\mathrm{Te}_{1}$ (второй набор) и $\mathrm{Te}_{2}-\mathrm{Te}_{2}$ (третий набор). Они располагаются в плоскостях, параллельных плоскости гексагональных осей $(a, b)$ и перпендикулярных оси $c$. Каждый из атомов имеет по 3 ближайших соседних атома и соответственно 3 критические точки типа bond. Первые два набора критических точек типа bond в структуре $\mathrm{Sb}_{2} \mathrm{Te}_{3}$ характеризуют связь между атомами внутри квинтета атомных слоев, в то время как третий набор критических точек типа bond определяет параметры существенно более слабой связи между квинтетами. Распределение электронной плотности в трех плоскостях $\mathrm{Sb}_{2} \mathrm{Te}_{3}$, в каждой из которых содержится по три критические точки типа bond, представлено на рис. 5. На рис. $5, a-c$ показано распределение полной



Рис. 4. Кристаллическая структура соединения $\mathrm{Sb}_{2} \mathrm{Te}_{3}$ в гексагональных координатных осях. 


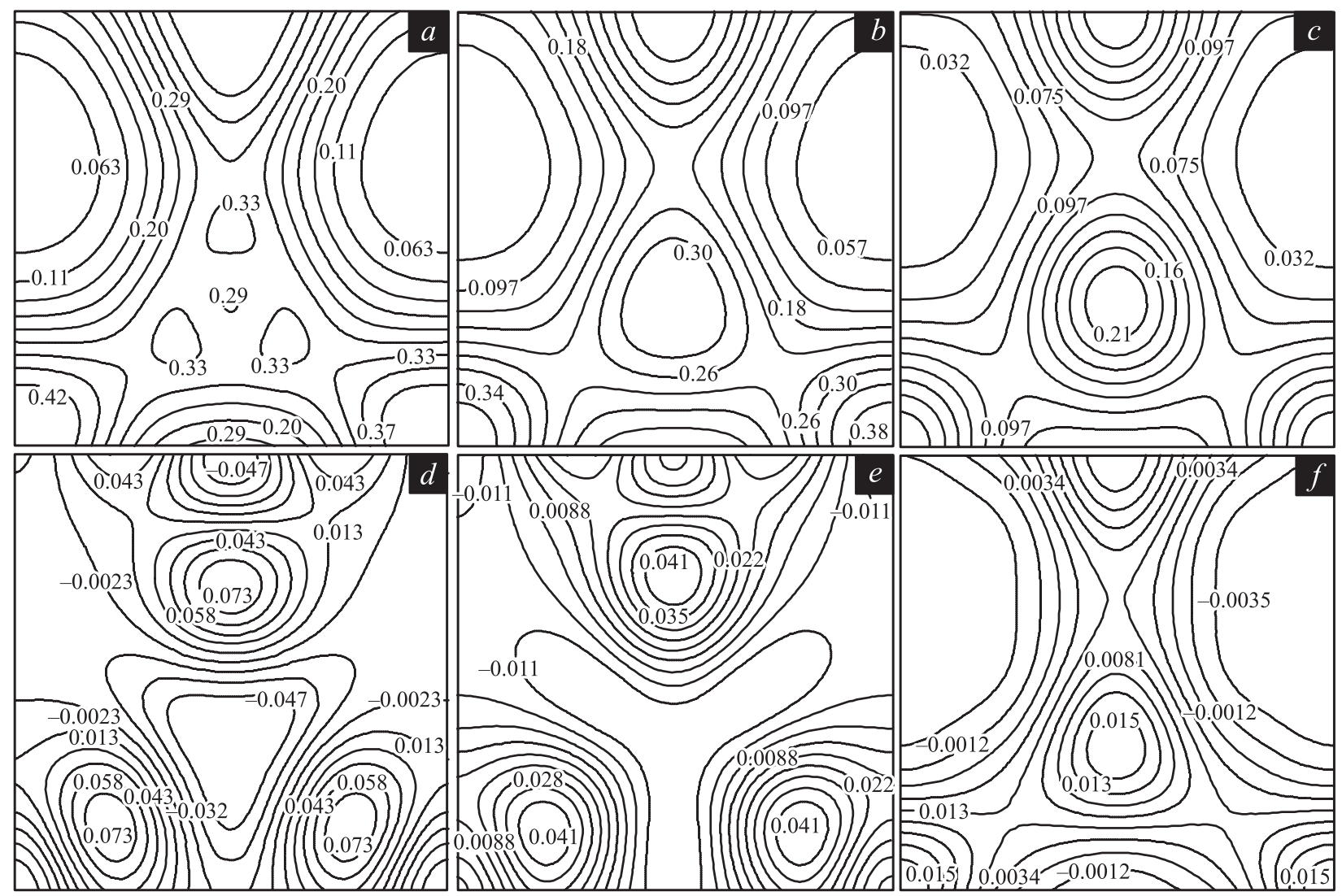

Рис. 5. Линии уровня распределения электронной плотности в плоскостях кристаллической структуры соединения $\mathrm{Sb}_{2} \mathrm{Te}_{3}$, содержащих критические точки типа bond. $a, b$ и $c-$ полная электронная плотность, $d, e$ и $f-$ электронная плотность, полученная методом CDD.

электронной плотности и на рис. $5, d-f-$ распределение электронной плотности от $s$ - и $p$-электронов атомов $\mathrm{Sb}$ и Те, полученное методом CDD. Рис. 5, $a$ и $d$ представляет распределение электронной плотности для первого набора критических точек типа bond, рис. 5, $b$ и $e$ - для второго набора и рис. 5, $c$ и $f$ - для третьего набора. Плоскости, содержащие критические точки типа bond, проведены параллельно плоскости $(a, b)$ системы координат в гексагональных осях на высоте 0.574 с для первой тройки идентичных критических точек типа bond, на высоте 0.632 с — для второй тройки и 0.167 c — для третьей тройки критических точек типа bond в pacпределении электронной плотности в кристаллической структуре $\mathrm{Sb}_{2} \mathrm{Te}_{3}$. На рис. 5, $a-c$ максимумы в распределении электронной плотности в плоскостях рисунка происходят от атомов, лежащих выше и ниже плоскостей, в которых располагаются критические точки типа bond. На рис. $5, d$ и $e$, полученных методом $\mathrm{CDD}$, отчетливо видны три критические точки в виде максимумов в распределении электронной плотности. Расположение линий уровня электронной плотности на рис. $5, f$ заметно отличается от такового на рис. $5, d$ и $e$. Видно, что в данной плоскости нет явно выраженных максимумов электронной плотности, обеспечивающей связь между квинтетами в структуре $\mathrm{Sb}_{2} \mathrm{Te}_{3}$. Данная плоскость является плоскостью скола для кристалла. Тем не менее из табл. 2 и рис. 3 можно сделать вывод, что связь между квинтетами из слоев атомов в структуре $\mathrm{Sb}_{2} \mathrm{Te}_{3}$ не является ван-дер-ваальсовой, как предполагалось в значительной части работ, посвященных исследованию электронной структуры $\mathrm{Sb}_{2} \mathrm{Te}_{3}$ и $\mathrm{Bi}_{2} \mathrm{Te}_{3}$, a, скорее, имеет признаки, характерные для полуметалла.

\section{4. Заключение}

Расчеты электронной зонной структуры полупроводников $\mathrm{Ge}$ и $\mathrm{Te}$, полуметалла $\mathrm{Sb}$ и соединения $\mathrm{Sb}_{2} \mathrm{Te}_{3}$, проведенные с помощью программного комплекса WIEN2k, основанного на методе функционала электронной плотности, позволили найти параметры критических точек в распределении электронной плотности для указанных веществ. Показано, что химическая связь в Те и $\mathrm{Sb}_{2} \mathrm{Te}_{3}$ имеет существенно меньшую степень ковалентности, чем в $\mathrm{Ge}$, и близка по характеру к связи в полуметаллической сурьме. Найденные особенности в распределении электронной плотности в кристаллической структуре соединения $\mathrm{Sb}_{2} \mathrm{Te}_{3}$ могут быть использованы для объяснения необычных свойств класса псевдобинарных сплавов $(\mathrm{GeTe})_{m}-\left(\mathrm{Sb}_{2} \mathrm{Te}_{3}\right)_{n}$, к которому принадлежит $\mathrm{Sb}_{2} \mathrm{Te}_{3}$, а также служить признаком для поиска новых веществ, обладающих свойствами РСМ. 
Авторы выражают благодарность И.А. Ткаченко за помощь в настройке пакета программ WIEN2k на многоцелевом вычислительном комплексе НИЦ „Курчатовский институт“ и Е.А. Марьину за помощь в подготовке иллюстраций.

\section{Список литературы}

[1] C. Wood. Rep. Prog. Phys. 51, 459 (1988).

[2] D. Hsieh, Y. Xia, D. Qian, L. Wray, J.H. Dil, J. Osterwalder, L. Patthey, A,V. Fedorov, H. Lin, A. Bansil, D. Grauer, Y.S. Hor, R.J. Cava, M.Z. Hasan. Phys. Rev. Lett. 103, 146401 (2009).

[3] M. Wuttig, N. Yamada. Nature Mater. 6, 824 (2007).

[4] T. Siegrist, P. Merkelbach, M. Wuttig. Ann. Rev. Condens. Matter Phys. 3, 215 (2012).

[5] V.L. Deringer, R. Dronkowski, M. Wuttig. Adv. Funct. Mater. 25, 6343 (2015).

[6] N. Yamada, E. Ohno, K. Nishiuchi, N. Akahira, M. Takao. J. Appl. Phys. 69, 2849 (1991).

[7] J.H. Coombs, A.P.J.M. Jongenelis, W. van Es-Spiekman, B.A. Jacobs. J. Appl. Phys. 78, 4906 (1995).

[8] M.H. Lankhost, B.W. Ketelaars, R.A. Wolters. Nature Mater. 4, 347 (2005).

[9] F. Xiong, A.D. Liao, D. Estrada, E. Pop. Science 232, 568 (2011).

[10] G. Lucovsky, R.M. White. Phys. Rev. B 8, 660 (1973).

[11] K. Shportko, S. Kremers, M. Woda, D. Lencer, J. Robertson, M. Wuttig. Nature Mater. 7, 653 (2008).

[12] L. Pauling. The Nature of the Chemical Bond. $3^{\text {rd }}$ ed. Cornell U.P., Ithaca, N.Y. (1960).

[13] P. Hohenberg, W. Kohn. Phys. Rev. 136, B864 (1964).

[14] W. Kohn, L.J. Sham. Phys. Rev. 140, A1133 (1965).

[15] W. Wełnic, A. Pamungkas, R. Detemple, C. Steimer, S. Blügel, M. Wuttig. Nature Mater. 5, 56 (2006).

[16] M. Wuttig, D. Lüsebrink, D. Wamwangi, W. Wełnic, M. Gilleßen, R. Dronskowski. Nature Mater. 6, 122 (2007).

[17] A.V. Kolobov, M. Krbal, P. Fons, J. Tominaga, T. Uruga. Nature Chem. 3, 311 (2011).

[18] A.V. Kolobov, P. Fons, J. Tominaga, S.R. Ovshinsky. Phys. Rev. B 87, 165206 (2013).

[19] A.V. Kolobov, P. Fons, J. Tominaga, M. Hase. J. Phys. Chem. C 118, 10248 (2014).

[20] R.F.W. Bader. In: Atoms in Molecules: A Quantum Theory. Int. Series of Monographs on Chemistry 22. Oxford Science Publications, Oxford (1990).

[21] C. Gatti. Z. Kristallogr. 220, 399 (2005).

[22] The Quantum Theory of Atoms in Molecules. From Solid State to DNA and Drug Design / Ed. C.F. Matta, R.J. Boyd. WILEY-VCH Verlag GmbH\&Co. KGaA, Weinheim (2007).

[23] P. Blaha, K. Schwarz, G.K.H. Madsen, D. Kvasnicka, J. Luitz. WIEN2k, An Augmented Plane Wave + Local Orbitals Program for Calculating Crystal Properties (Karlheinz Schwarz, Techn. Universität Wien, Austria) (2001). ISBN 3-9501031-1-2.

[24] F. Tran, P. Blaha. Phys. Rev. Lett. 102, 226401 (2009).

[25] H. Dixit, R. Saniz, S. Cottenier, D. Lamoen, B. Patroens. J. Phys.: Condens. Matter 24, 205503 (2012).

[26] http://computing.kiae.ru/

[27] A. Otero-de-la-Roza, E.R. Johnson, V. Luaña. Comp. Phys. Commun. 185, 1007 (2014).
[28] J.F.C. Baker, M. Hart. Acta Cryst. A 31, 364 (1975).

[29] C.S. Barrett, P. Cucka, K. Haefner. Acta Cryst. 16, 451 (1962).

[30] R. Keller, W.B. Holzapfel, H. Schulz. Phys. Rev. B 16, 4404 (1977).

[31] T.L. Anderson, H.B. Krause. Acta Cryst. B 30, 1307 (1974).

[32] G.G. Macfarlane, T.P. McLean, J.E. Quarrington, V. Roberts. Phys. Rev. 108, 1377 (1957).

[33] P. Grosse. Springer Tracts in Modern Physics. V. 48. SpringerVerlag, Berlin (1969).

[34] R. Sehr, L.R. Testardi. J. Phys. Chem. Solids 23, 1219 (1962).

[35] International Tables for Crystallography. Volume A. Spacegroup symmetry / Ed. Th. Hahn. Springer (2005).

[36] M. Morse, S.S. Cairns. In: Critical point theory in global analysis and differential geometry. Academic Press, N.Y. (1969).

[37] J.D. Joannopoulos, M. Schlütter, M.L. Cohen. Phys. Rev. B 11, 2186 (1975).

[38] G. Wang, T. Cagin. Phys. Rev. B 76, 075201 (2007). 